\title{
Systèmes Economiques Complexes et Vulnérabilité : Notes sur la Naissance de l'Economie de Service
}

\author{
par Orio Giarini*
}

Nous vivons actuellement, à l'échelle planétaire, une phase de transition structurelle profonde qui signe la fin de la révolution industrielle classique et ouvre la voie pour une nouvelle "économie de service".

Cette évolution repose à la fois sur une modification des facteurs économiques qui conditionnent la formation de la richesse, ainsi que sur un changement des instruments culturels et théoriques qui conditionnent la perception et les méthodes d'action vis-à-vis de l'économie.

Dans ce contexte, la notion de complexité joue un rôle primordial.

\section{1. l'Evolution de l'Economie Industrielle : du Simple au Complexe}

La révolution industrielle moderne a commencé en Angleterre vers le milieu du XVIII ${ }^{e}$ siècle par la diffusion de l'activité manufacturière fondée sur un certain nombre d'innovations spécifiques :

- pour mieux exploiter l'énergie produite par la machine à vapeur statique, capable d'alimenter plusieurs métiers à tisser, il convenait de réunir cet outil de production dans un lieu spécifique: la fabrique. La production textile qui, jusqu'alors, avait été un appendice des activités agricoles, devenait le premier grand secteur industriel autonome fondé sur une logistique de production propre;

- le capital nécessaire pour mettre sur pied une telle activité était alors, par rapport aux temps modernes, assez limité;

- la nouvelle production industrielle en usine permettait certes une augmentation importante de productivité, mais cette dernière n'était encore qu'un phénomène très limité par rapport à ce que nous connaissons aujourd'hui;

— la technologie elle-même était en fait très simple, à la portée des "bons ingénieurs" de l'époque, sans que personne ne soupçonne encore la possibilité de faire recours à la recherche scientifique.

* Professeur Associé d'Economie de Service à l'Institut Universitaire d'Etudes Européennes, Université de Genève. 
Dans cette première phase de la révolution industrielle, toute l'attention était portée sur la possibilité de produire plus, dans un milieu relativement spécialisé. La plupart des fonctions relatives à l'activité des productions modernes, que nous appelons "services", existaient encore à l'état indifférencié. Ce sont la spécialisation et l'élargissement des marchés qui allaient développer un système de plus en plus complexe à la fois dans un sens vertical et horizontal.

La complexification verticale est celle qui concerne la multiplication des étapes de transformation des matières premières en produit final, tandis que la complexification horizontale concerne les développements de toutes les activités de service (transport, stockage, financement, etc...), qui accompagnent et soutiennent l'activité de production elle-même.

Parallèlement à ce processus de spécialisation et de complexification verticale du système de production dans la révolution industrielle, il y a toute une autre série d'activités de soutien, qui ne prennent de l'importance qu'à partir d'un stade avancé d'industrialisation. Il s'agit là de toutes les fonctions qui sont normalement regroupées sous le nom d'activités de service, telles que stockage, recherche, formation, financement, vente et marketing, services de sécurité, recyclage des déchets, etc... Au début de la révolution industrielle, toutes ces fonctions existaient naturellement déjà, mais sous une forme indifférenciée: lorsque nous sommes en présence d'une petite production de type artisanal, les problèmes de stockage, de distribution et d'élimination des déchets existent, mais ils sont d'une importance secondaire et ils ne deviennent pas à proprement parler un "métier". Le passage d'une production textile pulvérisé entre des dizaines et des centaines de milliers de petites unités telle qu'elle existait en Europe au XVIII' siècle, aux grosses concentrations de production que nous connaissons aujourd'hui, a fait émerger la nécessité, de gérer professionnellement un nombre croissant de "services", dont voici quelques exemples:

- plus la dimension et la spécialisation augmentent, plus il faut faire en sorte que les flux des matériaux en entrée et en sortie puissent se faire sans accroc. Une petite boulangerie peut à la limite se procurer la farine pour produire son pain chaque jour, tandis qu'une fabrique de fibres synthétiques doit avoir prévu très longtemps à l'avance les flux des matières premières qui lui permettront de fonctionner;

- de même, au niveau de l'activité capitaliste de financement: au début de la révolution industrielle dominait la famille de l'individu riche qui était sa propre banque. Par la suite, au cours du siècle dernier, se sont développées les sociétés par action, vu la nécessité de cumuler les ressources financières de plusieurs individus. C'est seulement vers la fin du siècle dernier que les banques ont commencé à développer leur fonction de collecte de l'épargne pour l'investissement industriel;

- elles ont atteint aujourd'hui un niveau d'activité planétaire gigantesque au point que pour les projets d'investissements les plus importants, il y a aussi des consortium de banques au niveau mondial;

- la fonction de la recherche et du développement n'est devenue une fonction formalisée, c'est-à-dire un métier, qu'à partir des années 1930 (alors que l'utilisation consciente et volontariste de la recherche et surtout des résultats de la recherche fondamentale, a commencé vers le début du siècle). C'est en effet à cette époque que les laboratoires 
internes aux compagnies industrielles ainsi que les grands centres indépendants de recherche sous contrat prennent leur grand essor. Avant cette date, il ne s'agissait que de petites activités sporadiques et dont l'importance budgétaire dans l'industrie était tout à fait secondaire sinon complètement ignorée.

Les performances des nouvelles technologies requièrent avec le temps le développement de nouveaux "métiers", visant à controler l'accumulation des déchets ainsi que les aspects dangereux de certaines productions. Ce dernier aspect est devenu particulièrement aigü au cours des vingts dernières années, bien qu'il ait toujours existé à côté de chaque systèmes de production, mais sous une forme "indifférenciée" et avec des conséquences assez limitées.

Toutes ces activités de service qui accompagnent les systèmes de production, représentent aujourd'hui entre $70 \%$ et $80 \%$ du coût de tous les produits finals que nous utilisons.

Dans certains cas, ces activités de service représentent des fonctions à l'intérieur des compagnies industrielles et dans d'autres elles font l'objet d'une activité autonome, par des firmes spécifiques.

On peut donc affirmer qu'aujourd'hui l'importance prioritaire est dans l'économie des activités de service: l'importance quantitative de la production industrielle au sens strict n'est que seconde bien que naturellement toujours aussi essentielle.

La grande différence entre la structure de l'économie contemporaine aujourd'hui et celle de la révolution industrielle classique est que les activités de service sont actuellement non seulement quantitativement plus importantes, mais aussi essentielles pour rendre les activités de production utilisables. Au cours de la première révolution industrielle, les activités de service étaient certes toujours utiles, mais pas toujours indispensables ou essentielles.

A l'époque de Keynes, on pouvait encore mettre l'accent sur une politique visant à stimuler la production (l'offre) par la demande; aujourd'hui aucune production n'échappe à la nécessité d'être portée et soutenue par une infrastructure de service adéquate. "Services" et "production" sont désormais sur un pied d'égalité. Le bon fonctionnement de l'offre dépend de plus en plus d'une bonne infrastructure et d'une bonne politique des services.

Il y a toutefois une troisième dimension du processus de complexification qui, aujourd'hui, est encore plus important. Il s'agit du fait qu'avec la multiplication des étapes dans le processus de production et de distribution, il s'est avéré de plus en plus nécessaire de coordonner toutes ces activités séparées. En d'autres mots, nous n'avons pas seulement assisté à un développement progressif de la spécialisation dans un nombre croissant de domaines, mais aussi à son corollaire, c'est-à-dire à l'augmentation des problèmes de coordination et d'organisation entre toutes les parties du système.

Il s'agit ici d'un processus de complexification horizontal pour faire face justement aux problèmes d'information et de coordination qui accompagnent nécessairement tout processus de diversification de la spécialisation. L'économie moderne est ainsi devenue un système ou un réseau de plus en plus complexe. 
Certes, cette complexité est encore tout a fait relative. Si on la compare avec celle des systèmes biologiques, on pourrait même considérer qu'il s'agit encore là d'un système assez rudimentaire et embryonnaire. Mais c'est en tout cas en tant que réseau de plus en plus complexe, avec des lignes de communication verticales et horizontales qu'il faut comprendre l'économie moderne. Un réseau dans lequel les différentes parties doivent pouvoir s'intégrer et où les problèmes d'information et d'organisation du système lui-même sont devenus de plus en plus importants.

C'est dans cette perspective que nous pouvons aussi mieux apprécier la contribution de l'évolution technologique contemporaine: les nouvelles technologies ont surtout un impact au niveau des systèmes de communication et d'organisation de l'information. C'est exactement ce qu'il faut pour mieux gérer l'évolution de l'économie contemporaine. Et tout cela est très différent de l'orientation prédominante de la technologie au cours de la révolution industrielle classique, où il s'agissait surtout d'approfondir et d'améliorer les stades de production et de transformation des matériaux et des produits.

On peut donc définir l'économie contemporaine comme économie de service de la façon suivante: situation dans laquelle les ressources (ou facteurs de production) sont utilisées dans des fonctions de service dont une partie croissante représente des fonctions de stockage, transmission, élaboration et intégration des différentes informations.

Ces dernières fonctions ont d'ailleurs commencé à se développer d'une façon considérable depuis plusieurs décennies: elles ont donné lieu au développement de ce que l'on appelle les fonctions administratives et bureaucratiques, dans tous les domaines. Jusqu'aux années 1960, on attendait de l'innovation technologique ce que cette dernière avait fourni pendant deux siècles, c'est-à-dire de l'innovation fondamentale et révolutionnaire au niveau des systèmes de production. On s'était fait à l'idée que le secteur des services, au sens large, était un secteur à basse productivité servant de réservoir pour absorber les excédents de main d'œuvre qui ne pouvaient pas trouver d'emplois dans les secteurs primaire et secondaire. Or, le sens précis de l'ainsi-dite crise économique que nous vivons depuis une douzaines d'années, réside justement dans le fait que l'essentiel des innovations et des possibilités de développer la richesse économique s'est déplacé du côté des activités de service, dans le cadre d'un système de plus en plus complexe.

\section{La Nouvelle Economie de Service: I'Economie du Complexe}

La complexité du système économique contemporain, compte tenu aussi du fait qu'il est devenu, ces dernières décennies, véritablement planétaire, représente indubitablement un défi et une chance capitale dans la voie du progrès de l'humain. Cette complexité appelle - nous insistons - une révision de l'analyse économique qui puisse intégrer les flux que le système de production industrielle entretient avec les activités de service ainsi qu'avec le système non-monétarisé. Du bon fonctionnement de ces deux autres dimensions au système de production dépend en grande partie la possibilité d'envisager d'une façon positive le développement de la richesse du monde.

Gérer la complexité demande le développement de nouvelles aptitudes et de nouvelles théories ainsi que de méthodes de gestion vis-à-vis de deux problèmes-clé: la vulnérabilité et l'incertitude. 


\subsection{La gestion de la vulnérabilité}

En ce qui concerne la vulnérabilité, celle-ci concerne avant tout les capacités qu'ont les différents systèmes complexes de fonctionner sans accroc. Il est évident que des ressources doivent être convoyées vers la gestion de la vulnérabilité et que cette dernière est, dans un système complexe, un problème plus difficile à résoudre que dans un système simple. Il suffit pour cela d'étudier l'évolution des activités des compagnies d'assurances depuis ces deux dernières décennies. Il n'est pas sans intérêt de remarquer que pendant les années 70 , le chiffre d'affaires des compagnies d'assurances dans le monde a continué à se développer à un rythme annuel moyen réel d'environ $5 \%$ par an, tandis que le taux de croissance économique réel est descendu autours de $2 \%$. La théorie économique traditionnelle aurait prédit que les activités des assurances auraient ralenti encore plus que l'activité économique générale. Cette prédiction aurait été conforme à la loi d'Engel, selon qui le besoin d'assurances n'intervient qu'après que les besoins primaires aient été satisfaits : ceci est conforme à la théorie selon laquelle les activités de services sont tout à fait secondaires dans le développement économique. Mais ceci montre aussi à quel point l'analyse empirique de l'économie traditionnelle n'a pas relevé le fait que parallèlement au développement technologique des dernières décennies, les systèmes sont devenus de plus en plus complexes et vulnérables: d'où la nécessité de s'assurer qu'ils fonctionnent le mieux possible et de prévenir au maximum toute conséquence négative. D'ailleurs ce ne sont pas seulement les frais d'assurance qui ont augmenté plus que proportionnellement dans l'ensemble de l'économie, mais aussi les frais de prévention et de sécurité qui sont liés justement à l'augmentation de la complexité d'une part et à la nature même du développement technologique dans certains secteurs, d'autre part.

Le marché même des risques industriels dans l'assurance est un exemple de ce changement; tandis que dans un passé proche le problème essentiel des compagnies d'assurances était d'assurer la valeur des biens qui pouvaient être détruits (une maison, une usine, une machine), depuis quelque temps ce sont justement les dommages indirects provoqués par le fonctionnement ou le mauvais fonctionnement d'un système qui deviennent de plus en plus importants. Ces faits sont significatifs de l'émergence d'une économie de la vulnérabilité, qui est l'aspect concret du "management" du complexe. On gère de moins en moins de machines individuelles qui fonctionnent ou qui ne fonctionnent pas, mais de plus en plus de systèmes interdépendants et interconnectés qu'il faut organiser de façon à pouvoir contrôler au mieux la vulnérabilité. Une des clés principales pour le développement de l'économie et de la pensée économique de ces prochaines décennies sera justement celle d'optimiser la gestion de la vulnérabilité sous toutes ses formes, à la fois micro- et macro-économiques.

\subsection{Les défis de l'incertitude}

Ce changement dans les faits doit correspondre aussi à un changement dans les théories et dans les perceptions, et notamment dans la perception du temps. Dès que le temps est pris pour ce qu'il est, c'est-à-dire comme un flux continu qui ne peut être arrêté qu'artificiellement par une photographie, les notions d'incertitude et de déséquilibre remplacent celles de certitude et d'équilibre qui dominent encore la théorie économique. Il s'agit naturellement d'une incertitude qui peut être conditionnée à la fois par la volonté, le poids des tendances lourdes et deterministes, et l'irruption d'événements aléatoires. Le mélange de ces trois facteurs ne peut en aucune manière permettre de revenir à des théories prônant 
l'inéluctabilité ou le déterminisme historique absolu, ces legs de l'illuminisme occidental qui sapent aujourd'hui les possibilités du monde de demain. Aucune entreprise humaine, que ce soit celle d'un compositeur d'opéras ou d'un inventeur de machines ou d'un producteur d'automobiles n'a jamais commencé sinon dans un état d'extrême incertitude. Ce n'est qu'avec le temps que certaines initiatives peuvent émerger et s'inscrire dans les faits. D'où aussi la nécessité de mieux comprendre au niveau des faits économiques et sociaux les problèmes d'inertie, et non seulement les problèmes de probabilité, d'incertitude voire d'indétermination.

Ceci nous amène aussi à contredire les fondements mêmes du principe d'équilibre entre offre et demande tel qu'il est conçu actuellement par l'économique. Les courbes d'offre et de demande qui se rencontrent dans les graphiques dans un temps indéterminé, ont dans la pratique un comportement temporel bien différent. Toute décision de production intervient toujours bien avant toute décision d'achat ou d'utilisation du produit ou service disponible. Les activités de production en économie, comme dans toutes les formes biologiques, sont des activités multiples, basées sur l'incertain et, au mieux, sur une certaine probabilité de réussite. La concordance entre offre et demande dans l'équilibre général des économistes, masque plutôt l'essentiel du phénomène: c'est-à-dire que pour une pluralité de productions incertaines et qui cherchent à obtenir des résultats, il y a ensuite un processus de sélection, opéré par la "demande". Cette dernière permet de choisir, de sanctionner les activités de production qui ont une valeur quelconque, qu'il s'agisse de valeurs matérielles ou non. Mais la demande existante à chaque instant n'est qu'un parmi les différents signes et facteurs qui stimulent la production.

Sur ce point les économistes classiques qui insistaient beaucoup sur la valeur de l'offre (de la production), avaient raison: l'essentiel de toute activité vivante est de produire. Nous-mêmes, nous sommes essentiellement ce que nous faisons. Et, à la limite, l'ambition de la civilisation humaine consiste à pouvoir se produire à un niveau de conscience de plus en plus élargi. La demande est une opération d'élagage, de sélection, qui intervient essentiellement ex-post. Cette fonction de sélection est essentielle, mais surtout pour permettre qu'il puisse y avoir d'autres et nouvelles productions, qui elles, seront toutes - au départ incertaines.

Les économistes néo-classiques et keynesiens, se sont laissés entraîner par l'idée que la notion de valeur économique est essentiellement ancrée à l'activité de la demande (nous saurions alors surtout ce que nous consommons). Il s'agit ici peut-être d'un choix moral, mais il nous semble que dans le cas de Mozart comme dans celui de Henri Ford, l'acte essentiel est celui de quelqu'un qui prend le risque de produire: grâce à des facteurs qui ne sont jamais tous prévisibles, certaines productions rencontrent une demande favorable. Il faut donc à notre avis que les bases de l'économie de l'avenir puissent retrouver leurs véritables dimensions philosophiques et peut-être morales au niveau d'une "économie de l'offre".

La complexité, la vulnérabilité, l'incertitude et le temps réel sont ensuite des conditions opérationnelles d'une certaine volonté d'être et de devenir.

Dans cette perspective, l'acceptation de la complexité et de l'incertitude, c'est la volonté de comprendre et d'agir, en prenant des risques qui resteront inévitables tant qu'il y aura vie. Mais c'est ainsi que la vie peut continuer. On ne peut exister qu'en assumant l'incertain. 


\section{Economie de Service et Gestion des Risques}

Au cours de la Révolution Industrielle classique, le seul type de risque pris en considération par les économistes (et ceci surtout au niveau sociologique et micro-économique) est celui de l'entrepreneur. Les risques du type assurable (risques purs), n'ont eu droit dans le manuel d'économie, au plus, qu'à quelques notes en bas de page.

Dans l'économie de service, il est probable que l'on aboutira à une reconnaissance généralisée de l'importance stratégique pour l'économie de la gestion des risques purs. Ce sera l'aboutissement d'une longue période de gestation qui a vu d'abord la naissance de la notion de Risk Management et sa prise en considération officielle, de plus en plus, à la fois par les assurances et par les industries. En fait, la notion de Risk Management est l'indice d'un phénomène économique beaucoup plus fondamental: l'entrée des risques de types assurables au niveau des préoccupations stratégiques de la direction générale de toute entreprise économique.

Dans l'économie de service la notion de risque finira probablement, tôt ou tard, par englober d'une part les risques d'entrepreneurs traditionnels et d'autre part les risques de types assurables. Le fait que les deux soient considérés de plus en plus au niveau des décisions importantes et stratégiques, ne veut pas dire du tout qu'il y aura confusion entre les deux notions: il y aura toujours une ligne de démarcation très nette entre les deux, constituée en particulier par la notion de "moral hazard". Mais il devient de plus en plus évident que la bonne gestion du risque d'entrepreneur dépend de la gestion judicieuse du risque pur et vice versa, pour pouvoir contrôler et mieux développer les bons résultats de toute entreprise économique. En ce qui concerne plus particulièrement le risk management des risques purs, il nous semble très probable que l'évolution déjà amorçée par un grand nombre de compagnies d'assurances pour répondre à la demande de couverture des niveaux de vulnérabilité va se poursuivre et s'approfondir.

Cet aspect peut être schématisé de la façon suivante :

\section{Tableau : Offre d'assurances et demande de couverture de risques}

Risque de niveau
catastrophique


L'un des principaux problèmes illustrés par le tableau est que la demande économique de couvrir un risque s'exprime en termes de niveau de vulnérabilité: quelle que soit la source ou l'origine du risque et du sinistre, la partie demande est intéressée par un contrôle ou une couverture adéquate de son niveau de vulnérabilité. Il s'agit d'identifier des niveaux d'homogénéité horizontale.

Il est aussi très important de réexaminer en profondeur ce que peut signifier la notion d'homogénéité des classes de risques (homogénéité verticale) lorsque:

- Aujourd'hui un risque industriel peut se référer simultanément à une couverture d'assurance qui peut être offerte pour différentes classes de risques. La production d'une usine chimique peut être interrompue et provoquer des pertes d'exploitation; cet arrêt de la production peut être lié à l'identification d'un produit qui engendrera probablement des sinistres lorsqu'il sera utilisé. Dans ce cas il s'agit d'une responsabilité civile découlant du produit. D'autre part ceci peut obliger le fabricant à engager des frais pour retirer ses produits du marché. Il est évident que dans une situation où un seul accident engendre des possibilités de pertes dans des directions aussi différentes, le traitement d'un tel problème sur le plan des assurances doit tendre à intégrer autant de classes de risques différentes que possible (dans ce cas les pertes d'exploitation, les procédures de rappel et la responsabilité civile du produit). Ce problème a été très clairement présenté dans une récente étude de l'Association de Genève, intitulée «Recall Practices» ${ }^{1}$ (le retrait des produits);

- la technologie est très fréquemment synonyme de spécialisation et lorsque l'on souhaite aujourd'hui couvrir par exemple les risques associés aux activités spatiales, l'on constate que chaque programme spatial particulier présente une vulnérabilité différente ${ }^{2}$

- la question de la période de référence par rapport aux différentes classes de risques et en particulier celles ayant des répercussions catastrophiques constitue un troisième aspect très important; en d'autres termes le problème est que pour reconstituer un ensemble utilisable de données sur les risques (un univers statistique gérable), il est de plus en plus nécessaire de prendre en considération comme unité de temps non seulement une année d'activité comme c'est la procédure normale dans le système économique, mais très fréquemment des périodes de cinq, dix, vingt ans ou plus.

Ceci est également très fréquemment le résultat de la spécialisation et de l'accroissement de la vulnérabilité technologique. Ce point très important a évidemment un rapport étroit avec les politiques fiscales. La question économique clé est ici de savoir quelle est la période de temps (combien d'années) adéquate pendant laquelle une activité économique doit être prise en considération. La possibilité de gérer rationnellement un problème économique en dépend.

\footnotetext{
1 Voir "Geneva Papers on Risk and Insurance" No 22, janvier 1982.

${ }^{2}$ Ce problème a été étudié par EUROSAT à Genève pour une recherche financée par l'Association de Genève. (Voir “Geneva Papers on Risk and Insurance” No 35, avril 1985).
} 
Nous sommes convaincus que lorsque la pensée économique sera plus avancée en matière d'économie de service, la prise en considération de l'unité de temps propre à chaque phénomène économique pour pouvoir le mieux gérer, devrait s'imposer de plus en plus. Il faut bien admettre que nous vivons encore dans une situation dans laquelle l'unité de temps privilégiée est très souvent l'année de douze mois, corset trop rigide que nous avons hérité en économie de l'âge des anciennes sociétés agricoles.

Il faut aussi bien souligner le fait que ce problème d'horizontalisation de la demande d'assurance ne concerne pas que le secteur industriel, mais qu'elle concerne toutes les formes de vulnérabilité y compris celles des individus, qui se traduisent par une demande de sécurité sociale, de retraite, d'assurance-vie et d'épargne privé. 